\title{
Compact Direct Flux Reconstruction for the Navier-Stokes Equations on Dynamic Meshes
}

\author{
Lai Wang*, Meilin $\mathrm{Yu}^{\dagger}$ \\ Department of Mechanical Engineering, \\ University of Maryland, Baltimore County, Baltimore, MD 21250
}

\begin{abstract}
In this study, the high-order discontinuous compact direct flux reconstruction (CDFR) method is used to solve the two-dimensional (2D) Navier-Stokes equations on quadrilateral unstructured dynamic meshes. Within a standard element, the CDFR method employs compact finite difference (FD) techniques to directly construct the nodal spatial derivatives on Gauss-Legendre solution points. In the procedure of constructing an arbitrary CDFR method, the spatial derivatives are approximated with local fluxes on solution points and common fluxes on element interfaces (flux points) in FD forms. No polynomial reconstruction needs to be employed explicitly. It is observed that the CDFR method is identical with the direct flux reconstruction (DFR) method and the nodal flux reconstruction-discontinuous Galerkin (FR-DG) method if Gauss-Legendre points are selected as solution points. For simulations with dynamic meshes, the geometric conservation law (GCL) has been incorporated into the Navier-Stokes equations. The performance of CDFR methods has been verified with various test cases, including the Euler vortex propagation on deformable meshes, and the Couette flow. Laminar flows of $M a=0.2$ over a static circular cylinder $(R e=100,185)$ and an oscillating circular cylinder $(R e=185)$ have been studied to demonstrate the capability of the solver developed in this study.
\end{abstract}

\section{Introduction}

High-order computational fluid dynamics (CFD) methods have gained much research interest in the last three decades due to their superior capability of resolving turbulence, aeroacoustics, and unsteady vortex dynamics, etc., over their low-order counterparts (see the comprehensive reviews ${ }^{1-3}$ ). Currently, there exist many high-order accurate CFD methods, including pointwise schemes, such as compact FD schemes ${ }^{4}$ and weighted essentially non-oscillatory (WENO) schemes, ${ }^{5,6}$ and element-wise schemes, such as discontinuous Galerkin (DG) schemes, ${ }^{7,8}$ spectral volume (SV) schemes, ${ }^{9}$ spectral difference (SD) schemes ${ }^{10,11}$ and flux reconstruction/correction procedure via reconstruction $(\mathrm{FR} / \mathrm{CPR})$ schemes. $^{12-15}$

Comparing with element-wise schemes, FD schemes are easier to construct in general, and can achieve high-order accuracy with fewer mesh points through special design (e.g., the implicit approach in compact $\mathrm{FD}^{4}$ ). However, FD schemes are usually designed for structured meshes, limiting their application in solving practical engineering problems with complex geometries. Besides, compact FD schemes cannot maintain the scheme properties globally. Near boundaries, reconstructions of inner schemes (referred to as numerical boundary schemes (NBSs) in Ref. 16) have to be carried out using either ghost points ${ }^{17}$ or biased schemes ${ }^{18}$. To overcome the

\footnotetext{
*Graduate student, AIAA Student Member, email: bx58858@umbc.edu

${ }^{\dagger}$ Assistant professor, AIAA Senior Member, email: mlyu@umbc.edu
} 
instability possibly introduced by NBSs, the summation by parts (SBP) property ${ }^{19-21}$ has been proposed as a genuine property to ensure the stability of FD schemes. Instead, element-wise schemes are compact in design, and can handle engineering flows over/through complex geometries with high-order accuracy. First developed by Huynh, ${ }^{12,13}$ the high-order FR/CPR method has demonstrated its capacity to handle complex flow problems on unstructured meshes. ${ }^{22}$ Correction functions are essential for original FR/CPR schemes. Recently, Romero et al. ${ }^{23,24}$ developed a simplified FR/CPR approach, i.e. the DFR scheme, which can obtain high-order accurate schemes without correction functions. Specifically, direct polynomial fitting involving all solution and flux points within an element is used. We recognize that no matter in the original FR/CPR or DFR, various types of polynomials need to be explicitly constructed and differentiated. These procedures could make them hard to implement.

The CDFR method ${ }^{25}$ utilizes compact FD techniques within an element to directly approximate the spatial derivatives on solution points. The final form of the spatial derivatives are expressed in FD forms. This method does not involves any polynomial reconstructions ${ }^{23}$ or correction functions. ${ }^{12}$ By casting the DFR method and FR-DG method in a similar FD form as that of the CDFR method, we observe that the CDFR method is identical with them if Gauss-Legendre points are employed as the solutions points. In this study, we extend the CDFR method to solve the Navier-Stokes equations on deformable meshes. The GCL is enforced analytically in the time-dependent transformation of the governing equations from the physical domain to the computational domain. ${ }^{26-28}$ Compared to solving an additional equation regarding the Jacobians of the coordinate mapping, ${ }^{29}$ the approach employed here integrates the GCL into the governing equations as a source term. An explicit strong-stability-preserving three-stage Runge-Kutta (SSPRK3) is employed for the time integration.

The remainder of this paper is organized as follows. In Section 2, we revisit the construction procedure of the CDFR method for the one dimensional (1D) conservation law. In Section 3 , a time-dependent coordinate transformation of the 2D Navier-Stokes equations from the physical domain to computational domain is presented. This transformation can facilitate the implementation of the CDFR method in a tensor product approach. Section 4 explains the strategy used to enforce GCL in numerical simulation with dynamic meshes. In Section 5, the accuracy performance of the CDFR method has been demonstrated through various numerical tests. The last section summarizes the current work.

\section{The Compact Direct Flux Reconstruction Method}

In this section, we revisit the construction procedure of the CDFR method for the 1D conservation law. In the CDFR method, the governing equation is projected onto a standard element $\xi \in[-1,1]$ and the Gauss-Legendre points are employed as the solution points. ${ }^{25}$ The compact FD techniques are adopted in the procedure to directly obtain the spatial derivatives of fluxes on the solution points. These spatial derivatives' approximations are obtained in FD forms.

\section{II.A. Construction procedure of the CDFR method}

Consider the 1D scalar conservation law expressed as

$$
u_{t}+f_{x}=0, x \in \Omega \text {. }
$$

The domain $\Omega$ is tessellated with elements $\Omega_{j}=\left\{x: x \in\left[x_{j-1 / 2}, x_{j+1 / 2}\right]\right\}, j=1,2, \ldots, N$. In this study, each element is projected onto the standard element $[-1,1]$. In terms of the coordinate transformation,

$$
f_{x}=f_{\xi} \xi_{x}, \xi_{x}=\Delta \xi / \Delta x, \text { and } \Delta \xi=2 .
$$

The CDFR method uses $k$ Gauss-Legendre points as the solution points and two element interfaces as the flux points to approximate the spatial derivatives on the solution points directly. 
Since the construction is conducted within the standard element, we drop the subscript " $\xi$ " in any $n$-th order spatial derivative $f_{\xi}^{(n)}$. The whole procedure is completed using compact FD approaches. This procedure does not involve any polynomial reconstructions that conventional FR/CPR methods and DFR methods need.

The Taylor expansion of the spatial derivative $f^{\prime}\left(\xi_{i}\right), i=0,1, \ldots, k+1$ on each solution points with respect to the element center $\xi=0$ can be expressed as

$$
\begin{gathered}
f^{\prime}\left(\xi_{i}\right)=f^{\prime}(0)+\left(\xi_{i}\right) f^{\prime \prime}(0)+\frac{\left(\xi_{i}\right)^{2}}{2 !} f^{\prime \prime \prime}(0)+\ldots+\frac{\left(\xi_{i}\right)^{k-1}}{(k-1) !} f^{(k)}(0)+O\left(\left(\xi_{i}\right)^{k}\right), \\
f\left(\xi_{i}\right)=f(0)+\left(\xi_{i}\right) f^{\prime}(0)+\frac{\left(\xi_{i}\right)^{2}}{2 !} f^{\prime \prime}(0)+\ldots+\frac{\left(\xi_{i}\right)^{k}}{(k) !} f^{(k)}(0)+O\left(\left(\xi_{i}\right)^{k+1}\right) .
\end{gathered}
$$

Herein, $\xi_{i}, i=1,2, \ldots, k$ are the Gauss-Legendre solution points and $\xi_{0}=-1, \xi_{k+1}=1$ are the left and right element interfaces respectively. Eq. (4) can be rewritten as

$$
\frac{1}{\Delta \xi} f\left(\xi_{i}\right)=\frac{1}{\Delta \xi}\left\{f(0)+\left(\xi_{i}\right) f^{\prime}(0)+\frac{\left(\xi_{i}\right)^{2}}{2 !} f^{\prime \prime}(0)+\ldots+\frac{\left(\xi_{i}\right)^{k}}{(k) !} f^{(k)}(0)\right\}+O\left(\left(\xi_{i}\right)^{k}\right) .
$$

Generally, for a CDFR method with $k$ solution points, $k$ compact FD discretization equations can be formulated $\mathrm{as}^{25}$

$$
\sum_{i=1}^{k} \alpha_{l i} f_{i}^{\prime}=\frac{1}{\Delta \xi} \sum_{j=l-1}^{l+1} \beta_{l(j+1)} f_{j}, l=1,2, \ldots, k
$$

to solve the $k$ unknowns, i.e. spatial derivatives $f^{\prime}$. Note that for the $l$-th $(l=1,2, \ldots, k)$ compact FD discretization, the stencil involved in the l.h.s. of Eq. (6) employs all the $k$ solution points while only involves three consecutive points, i.e., $\xi_{j}, j=l-1, l, l+1$ on the r.h.s. After substituting Taylor expansions Eq. (3) and Eq. (4) into Eq. (6), the coefficient of $f_{i}^{(n)}$ can be expressed as

$$
\frac{\xi_{i}^{n-1}}{(n-1) !}, i=1,2, \ldots, k ; n \geq 1
$$

for the l.h.s. and

$$
\frac{1}{\Delta \xi} \frac{\xi_{j}^{n}}{(n) !}, j=l-1, l, l+1 ; l=1,2, \ldots, k ; n \geq 0
$$

for the r.h.s. of the discretization Eq. (6), respectively. There are $k$ degrees of freedom on the l.h.s. of Eq. (6) and three degrees of freedom on the r.h.s. of Eq. (6). The CDFR method employs the restriction $\sum_{i=1}^{i=k} \alpha_{l i}=1$ and requires the coefficient of $f^{(n)}$ on each side of Eq. (6) to be consistent from 0 to $k+1$. Therefore, for each discretization Eq. (6), $k+3$ equations can be formulated to obtain the corresponding coefficients in $\boldsymbol{\alpha}$ and $\boldsymbol{\beta} . \boldsymbol{M}_{l} \boldsymbol{C}_{l}=\boldsymbol{B}$ is adopted here to denote these equation of the $l$-th compact discretization, which can be expressed as

$$
\left\{\begin{array}{l}
-\sum_{j=l-1}^{j=l+1} \beta_{l(j+1)}=0, \\
\sum_{i=1}^{i=k} \frac{\xi_{i}^{n-1}}{(n-1) !} \alpha_{l i}-\frac{1}{2} \sum_{j=l-1}^{j=l+1} \frac{\xi_{j}^{n}}{(n) !} \beta_{l(j+1)}=0, n=1,2, \ldots, k+1, \\
\sum_{i=1}^{i=k} \alpha_{l i}=1,
\end{array}\right.
$$

where $l=1,2, \ldots, k$. After solving $k$ linear systems in the form of Eq. (9), $\boldsymbol{\alpha}$ and $\boldsymbol{\beta}$ can be obtained. Hence, we can formulate the final discretization form for spatial derivatives as

$$
\boldsymbol{f}^{\prime}=\frac{1}{\Delta \xi} \boldsymbol{\alpha}^{-\mathbf{1}} \boldsymbol{\beta} \boldsymbol{f},
$$


where $\boldsymbol{f}=\left(f_{1}^{\prime}, f_{2}^{\prime}, \ldots, f_{k}^{\prime}\right)^{\top}$ and $\boldsymbol{f}=\left(f_{0}, f_{1}, f_{2}, \ldots, f_{k+1}\right)^{\top}$. Here, $f_{0}$ and $f_{k+1}$ are the common fluxes on the interfaces. Riemann solvers ${ }^{30}$ are used to calculate the common fluxes. Particularly, if $f=c u$ with $c$ a constant in Eq. (1)), the left common flux of the current element $\Omega_{j}$ can be expressed as

$$
f_{0}^{j}= \begin{cases}f_{k+1, l o c}^{j-1}, & \text { if } c>0 \\ f_{0, l o c}^{j}, & \text { if } c<0\end{cases}
$$

Since the common flux solver involves neighboring elements, we introduce superscript ' $j$ ' to denote the $j$-th element. Lagrange interpolation is used to obtain local fluxes $f_{0, l o c}^{j}$ and $f_{k+1, l o c}^{j}$ on interfaces as

$$
f_{0 / k+1, l o c}^{j}=\sum_{i=1}^{k}\left(\left(\prod_{l=1, l \neq i}^{k} \frac{\xi_{0 / k+1}-\xi_{l}}{\xi_{i}-\xi_{l}}\right) f_{i}^{j}\right) .
$$

A MATLAB symbolic calculation with extended precision (64 digits) is used to obtain these coefficients.

\section{II.B. Connection with the DFR method and FR-DG method}

The Taylor expansion approach is built on the premise that there exists a function which can be continuously differentiated. Eq. (10) shows that spatial flux derivatives on solution points within one element $\Omega_{j}$ can be discretely approximated by a combination of fluxes on solution points and interfacial flux points as

$$
f_{i}^{\prime}=\frac{1}{\Delta \xi} \sum_{j=1}^{k+2}\left(\boldsymbol{\alpha}^{-\mathbf{1}} \boldsymbol{\beta}\right)_{i j} f_{j-1}
$$

The scheme proposed in the present study implicitly constructs a flux polynomial spanning all solution points inside the element and flux points on the element interfaces.

The derivative of the flux polynomial of DFR with Gauss-Legendre solution points as developed in Ref. 23 can be expressed as

$$
f_{i}^{\prime}=\frac{1}{\Delta \xi} \sum_{j=1}^{k+1}\left(l_{D, j}^{\prime}\left(\xi_{i}\right) \Delta \xi\right) f_{j}
$$

where $l_{D, j}^{\prime}$ is the derivative of the Lagrange polynomial $l_{D, j}$ constructed from $\xi_{j}, j=0,1, \ldots, k+$ 1. The nodal FR-DG method with Gauss-Legendre solution points ${ }^{12}$ can also be formulated in a similar form as

$f_{i}^{\prime}=\frac{1}{\Delta \xi}\left\{\sum_{j=1}^{k}\left(\left[l_{C, j}^{\prime}\left(\xi_{i}\right)-l_{C, j}(-1) g_{L}^{\prime}\left(\xi_{i}\right)-l_{C, j}(1) g_{R, i}^{\prime}\left(\xi_{i}\right)\right] \Delta \xi\right) f_{j}+\left(g_{L}^{\prime}\left(\xi_{i}\right) \Delta \xi\right) f_{0}+\left(g_{R}^{\prime}\left(\xi_{i}\right) \Delta \xi\right) f_{k+1}\right\}$

where $g_{L}$ and $g_{R}$ are the left and right correction functions (e.g., Radau polynomials for FR-DG). Herein, the Lagrange polynomial $l_{C, j}$ is constructed from $\xi_{j}, j=1,2, \ldots, k$ without the two interface points $\xi_{0}$ and $\xi_{k+1}$. By numerically comparing the coefficients of $f_{i}, i=0,1, \ldots, k+1$, we observe that Eq. (13), Eq. (14), and Eq. (15) are identical with each other. Ref. 23 gives the analytical proof of the equality between Eq. (14) and Eq. (15).

In spite of the identity observed by comparing the coefficients of different schemes, the element-wise compact FD scheme proposed here shares a similarity with DFR to achieve highorder accuracy by making full use of all the solution and interfacial flux points. It directly approximates the spatial derivatives without constructing any flux polynomials that the DFR method needs or involving any correction functions that the original FR/CPR method requires. 


\section{The 2D Navier-Stokes Equations}

When the CDFR method is employed for multi-dimensional problems (2D problems with quadrilateral elements in this study), the spatial derivatives can be solved dimension by dimension. The compressible 2D Navier-Stokes equations can be written as

$$
\frac{\partial \boldsymbol{q}}{\partial t}+\frac{\partial \boldsymbol{f}}{\partial x}+\frac{\partial \boldsymbol{g}}{\partial y}=0
$$

where $\boldsymbol{q}=(\rho, \rho u, \rho v, E)^{T}$ are the conservative variables, $p=\rho R T, E=\frac{p}{\gamma-1}+\frac{1}{2} \rho\left(u^{2}+v^{2}\right), R$ is the ideal gas constant, and $\gamma$ is the specific heat ratio defined as $\gamma=C_{p} / C_{v}$. In this study, $\gamma$ is set as 1.4. The fluxes $\boldsymbol{f}$ and $\boldsymbol{g}$ consist of inviscid part and viscous part, which can be expressed as $\boldsymbol{f}=\boldsymbol{f}_{i n v}-\boldsymbol{f}_{v i s}$ and $\boldsymbol{g}=\boldsymbol{g}_{i n v}-\boldsymbol{g}_{v i s}$. The component forms of $\boldsymbol{f}_{i n v}, \boldsymbol{g}_{i n v}, \boldsymbol{f}_{v i s}$, and $\boldsymbol{g}_{v i s}$ can be found below,

$$
\begin{gathered}
\boldsymbol{f}_{i n v}=\left(\begin{array}{c}
\rho u \\
\rho u^{2}+p \\
\rho u v \\
(E+p) u
\end{array}\right), \boldsymbol{g}_{i n v}=\left(\begin{array}{c}
\rho v \\
\rho u v \\
\rho v^{2}+p \\
(E+p) v
\end{array}\right), \\
\boldsymbol{f}_{v i s}=\left(\begin{array}{c}
0 \\
0 \\
\tau_{x x} \\
\tau_{x y} \\
u \tau_{x x}+v \tau_{x y}+k \frac{\partial T}{\partial x}
\end{array}\right), \boldsymbol{g}_{v i s}=\left(\begin{array}{c}
\tau_{y y} \\
u \tau_{x y}+v \tau_{y y}+k \frac{\partial T}{\partial y}
\end{array}\right) .
\end{gathered}
$$

Herein $k=\frac{\mu C_{p}}{\operatorname{Pr}}, \operatorname{Pr}=0.72$ and $\mu$ is also treated as a constant in the present work. Viscous stresses are given by

$$
\left\{\begin{array}{l}
\tau_{x x}=\mu\left(\frac{4}{3} \frac{\partial u}{\partial x}-\frac{2}{3} \frac{\partial v}{\partial y}\right), \\
\tau_{y y}=\mu\left(\frac{4}{3} \frac{\partial v}{\partial y}-\frac{2}{3} \frac{\partial u}{\partial x}\right), \\
\tau_{x y}=\tau_{y x}=\mu\left(\frac{\partial u}{\partial y}+\frac{\partial v}{\partial x}\right) .
\end{array}\right.
$$

After transforming Eq. (16) from the physical space-time domain $(x, y, t)$ into the computational space-time domain $(\xi, \eta, \tau)$ we can get

$$
\frac{\partial \boldsymbol{Q}}{\partial \tau}+\frac{\partial \boldsymbol{F}}{\partial \xi}+\frac{\partial \boldsymbol{G}}{\partial \eta}=0
$$

where

$$
\left\{\begin{array}{l}
\boldsymbol{Q}=|\boldsymbol{J}| \boldsymbol{q}, \\
\boldsymbol{F}=|\boldsymbol{J}|\left(\boldsymbol{q} \xi_{t}+\boldsymbol{f} \xi_{x}+\boldsymbol{g} \xi_{y}\right), \\
\boldsymbol{G}=|\boldsymbol{J}|\left(\boldsymbol{q} \eta_{t}+\boldsymbol{f} \eta_{x}+\boldsymbol{g} \eta_{y}\right),
\end{array}\right.
$$

and

$$
\boldsymbol{J}=\frac{\partial(x, y, t)}{\partial(\xi, \eta, \tau)}
$$

Herein, $t=\tau$ and $(\xi, \eta) \in[-1,1] \times[-1,1]$ is the standard element as illustrated in Figure 1 . Therefore, ${ }^{27}$

$$
\boldsymbol{J}=\left(\begin{array}{ccc}
x_{\xi} & x_{\eta} & x_{\tau} \\
y_{\xi} & y_{\eta} & y_{\tau} \\
0 & 0 & 1
\end{array}\right) \text {, and }|\boldsymbol{J}|=x_{\xi} y_{\eta}-x_{\eta} y_{\xi} .
$$

Note that

$$
\left\{\begin{array}{l}
\xi_{t}=-\boldsymbol{v}_{g} \cdot \nabla \xi \\
\eta_{t}=-\boldsymbol{v}_{g} \cdot \nabla \eta
\end{array}\right.
$$


which give the information about the grid motion. On an interface of the quadrilateral elements, $\boldsymbol{F}$ and $\boldsymbol{G}$ in Eq. (21) can be rewritten as

$$
\left\{\begin{array}{l}
\boldsymbol{F}=|\boldsymbol{J}||\nabla \xi| \boldsymbol{f}_{\boldsymbol{n}} \operatorname{sign}(\boldsymbol{n} \cdot \nabla \xi) \\
\boldsymbol{G}=|\boldsymbol{J}||\nabla \eta| \boldsymbol{f}_{\boldsymbol{n}} \operatorname{sign}(\boldsymbol{n} \cdot \nabla \eta)
\end{array}\right.
$$

where

$$
|\nabla \xi|=\frac{1}{|\boldsymbol{J}|} \sqrt{x_{\eta}^{2}+y_{\eta}^{2}},|\nabla \eta|=\frac{1}{|\boldsymbol{J}|} \sqrt{x_{\xi}^{2}+y_{\xi}^{2}},
$$

and $\boldsymbol{n}=\left(n_{x}, n_{y}\right)$ is the unit outward-normal vector at the interface. Herein, $\boldsymbol{f}_{\boldsymbol{n}}$ is the flux in normal direction of the current interface. Note that common fluxes are always calculated in the physical domain. Therefore, $\boldsymbol{F}_{\text {com }}$ and $\boldsymbol{G}_{c o m}$ can be expressed as

$$
\left\{\begin{array}{l}
\boldsymbol{F}^{c o m}=|\boldsymbol{J} \| \nabla \xi| \boldsymbol{f}_{\boldsymbol{n}}^{c o m} \operatorname{sign}(\boldsymbol{n} \cdot \nabla \xi) \\
\boldsymbol{G}^{\text {com }}=|\boldsymbol{J}||\nabla \eta| \boldsymbol{f}_{\boldsymbol{n}}^{c o m} \operatorname{sign}(\boldsymbol{n} \cdot \nabla \eta)
\end{array},\right.
$$

where $\boldsymbol{f}_{\boldsymbol{n}}^{\text {com }}$ is the common flux. The inviscid part of $\boldsymbol{f}_{\boldsymbol{n}}^{\text {com }}$ including the flux caused by deforming grids is given as

$$
\boldsymbol{f}_{i n v, \boldsymbol{n}}^{c o m}=\frac{1}{2}\left(\boldsymbol{f}_{i n v, \boldsymbol{n}}^{L}+\boldsymbol{f}_{i n v, \boldsymbol{n}}^{R}-\boldsymbol{R}|\boldsymbol{\Lambda}| \boldsymbol{R}^{-1}\left(\boldsymbol{q}^{R}-\boldsymbol{q}^{L}\right)\right)
$$

where

$$
\boldsymbol{f}_{\text {inv }, \boldsymbol{n}}=\boldsymbol{f}_{\boldsymbol{i n \boldsymbol { v }}} n_{x}+\boldsymbol{g}_{\boldsymbol{i n \boldsymbol { v }}} n_{y}-\left(\boldsymbol{v}_{g} \cdot \boldsymbol{n}\right) \boldsymbol{q} .
$$

The subscripts ' $L$ ' and ' $R$ ' denote the left side and right side of the current interface, respectively. In our implementation, the ' $L$ ' side of the interface is always the current element. The Jacobian matrix $A$ of the inviscid fluxes with respect to conservative variables is

$$
\boldsymbol{A}=\frac{\partial \boldsymbol{f}}{\partial \boldsymbol{q}} n_{x}+\frac{\partial \boldsymbol{g}}{\partial \boldsymbol{q}} n_{y}-\boldsymbol{v}_{g} \cdot \nabla \boldsymbol{q},
$$

where $|\boldsymbol{\Lambda}|$ is the diagonal matrix with absolute values of eigenvalues and $\boldsymbol{R}$ is a matrix consisting of the corresponding eigenvectors. Herein, the Roe average ${ }^{30}$ is used to calculate $\boldsymbol{R}, \boldsymbol{R}^{-\mathbf{1}}$, and $|\Lambda|$.

For the calculation of the viscous part of the common flux, note that $\boldsymbol{f}_{\text {vis }}=\boldsymbol{f}_{\text {vis }}(\boldsymbol{q}, \nabla \boldsymbol{q})$ and $\boldsymbol{g}_{v i s}=\boldsymbol{g}_{v i s}(\boldsymbol{q}, \nabla \boldsymbol{q})$. The common viscous flux is obtained by taking average, expressed as

$$
\boldsymbol{f}_{v i s, \boldsymbol{n}}^{c o m}=\frac{1}{2}\left(\boldsymbol{f}_{v i s, \boldsymbol{n}}^{L}+\boldsymbol{f}_{v i s, \boldsymbol{n}}^{R}\right) \text {. }
$$

Applying the CDFR method to solve Eq. (20), we can use the spatial discretization to obtain $\frac{\partial \boldsymbol{F}}{\partial \xi}$ and $\frac{\partial \boldsymbol{G}}{\partial \eta}$ one by one. Thus, the extension of the CDFR method to solve the 2D Navier-Stokes equations is completed.

\section{The Geometric Conservation Law with the Explicit Time Stepping}

The GCL for the metrics of the transformation from the physical domain to the computational domain can be formulated as

$$
\left\{\begin{array}{l}
\frac{\partial}{\partial \xi}\left(|\boldsymbol{J}| \xi_{x}\right)+\frac{\partial}{\partial \xi}\left(|\boldsymbol{J}| \eta_{x}\right)=0, \\
\frac{\partial}{\partial \xi}\left(|\boldsymbol{J}| \xi_{y}\right)+\frac{\partial}{\partial \xi}\left(|\boldsymbol{J}| \eta_{y}\right)=0, \\
\frac{\partial|\boldsymbol{J}|}{\partial t}+\frac{\partial}{\partial \xi}\left(|\boldsymbol{J}| \xi_{t}\right)+\frac{\partial}{\partial \xi}\left(|\boldsymbol{J}| \eta_{t}\right)=0 .
\end{array}\right.
$$

The first two equations only depend on the accuracy of the spatial discretizations while the last one is related to the grid motion. If only a rigid motion is involved, $|\boldsymbol{J}|$ is independent of time. For deformable meshes, apply the chain rule to $\frac{\partial \boldsymbol{Q}}{\partial t}$ to obtain

$$
\frac{\partial \boldsymbol{Q}}{\partial t}=\frac{\partial|\boldsymbol{J}| \boldsymbol{q}}{\partial t}=|\boldsymbol{J}| \frac{\partial \boldsymbol{q}}{\partial t}+\boldsymbol{q} \frac{\partial|\boldsymbol{J}|}{\partial t}
$$




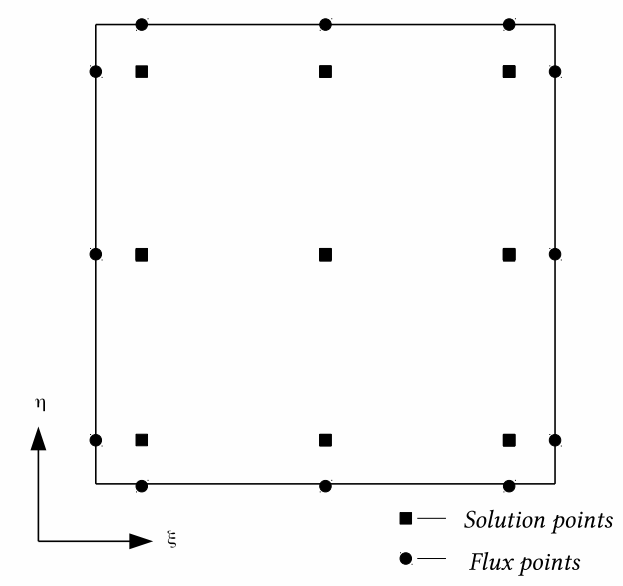

Figure 1. Illustration of solution points and flux points in a standard element for the 3rd order CDFR scheme.

By substituting Eq. (33) into Eq. (20), we can rewrite it as

$$
\frac{\partial \boldsymbol{q}}{\partial t}=\frac{1}{|\boldsymbol{J}|}\left\{-\left(\frac{\partial \boldsymbol{F}}{\partial \xi}+\frac{\partial \boldsymbol{G}}{\partial \eta}\right)+\boldsymbol{S}\right\}
$$

where $\boldsymbol{S}=\left[\frac{\partial}{\partial \xi}\left(|\boldsymbol{J}| \xi_{t}\right)+\frac{\partial}{\partial \xi}\left(|\boldsymbol{J}| \eta_{t}\right)\right]$. Thus, the GCL is enforced. In Refs. 29, the enforcement of GCL is achieved by employing the same time integration form for the Jacobian of the transformation as the conservative variables. An extra equation needs to be solved iteratively to enforce the GCL. In the present study, the ' $\boldsymbol{S}$ ' term regarded as a source term is added into the r.h.s. of Eq. (34) to balance the mesh deformation terms in $\boldsymbol{F}$ and $\boldsymbol{G} \cdot{ }^{26,27}$ For the calculation of term $\boldsymbol{S}$, the CDFR method is also adpoted.

An explicit strong-stability-preserving three-stage Runge-Kutta scheme ${ }^{31}$ is employed for the time marching. It can be expressed as

$$
\left\{\begin{array}{l}
\boldsymbol{q}^{(1)}=\boldsymbol{q}^{n}+\Delta t L\left(\boldsymbol{q}^{n}\right) \\
\boldsymbol{q}^{(2)}=\frac{3}{4} \boldsymbol{q}^{n}+\frac{1}{4} \boldsymbol{q}^{(1)}+\frac{1}{4} \Delta t L\left(\boldsymbol{q}^{(1)}\right) \\
\boldsymbol{q}^{n+1}=\frac{1}{3} \boldsymbol{q}^{n}+\frac{2}{3} \boldsymbol{q}^{(2)}+\frac{2}{3} \Delta t L\left(\boldsymbol{q}^{(2)}\right)
\end{array}\right.
$$

where $\boldsymbol{L}=\frac{1}{|\boldsymbol{J}|}\left\{-\left(\frac{\partial \boldsymbol{F}}{\partial \xi}+\frac{\partial \boldsymbol{G}}{\partial \eta}\right)+S\right\}$. In the present study, all unsteady tests are conducted with SSPRK3.

\section{Numerical Results}

In this section, several benchmark cases are simulated to verify the numerical accuracy of the CDFR methods for 2D problems. Both steady and unsteady cases are considered. The performance of CDFR with curved boundaries are also tested. The $L_{2}$ error is used for the accuracy study. Specifically, for any solution variable $s$ on the domain $\Omega$, the $L_{2}$ error is defined as

$$
E_{L_{2}}(s, \Omega)=\sqrt{\frac{\int_{\Omega}\left(s-s^{\text {exact }}\right)^{2} d V}{\int_{\Omega} 1 d V}} .
$$

\section{V.A. 2D Isentropic Euler Vortex Propagation}

The isentropic vortex propagation case depicts the superposition of an inviscid uniform flow and an irrotational vortex. The vortex can be regarded as a perturbation added into the uniform 
flow. The free stream flow is of $(p, u, v, T)=(1.0, \sqrt{\gamma}, \sqrt{\gamma}, 1.0)$. The ideal gas constant is set as $R=1.0$ for this case. The perturbation is defined as $^{32}$

$$
\left\{\begin{array}{l}
\delta u=-\frac{\alpha}{2 \pi}\left(y-y_{0}\right) e^{\phi\left(1-r^{2}\right)} \\
\delta v=\frac{\alpha}{2 \pi}\left(x-y_{0}\right) e^{\phi\left(1-r^{2}\right)} \\
\delta T=-\frac{\alpha^{2}(\gamma-1)}{16 \phi \gamma \pi^{2}}\left(y-y_{0}\right) e^{2 \phi\left(1-r^{2}\right)} \\
d S=0
\end{array}\right.
$$

where $\phi=\frac{1}{2}$ and $\alpha=5$ are parameters that define the vortex strength. $r=\left(x-x_{0}\right)^{2}+\left(y-y_{0}\right)^{2}$ is the distance to the center of the vortex $\left(x_{0}, y_{0}\right)=(5,5)$ at $t=0$. The periodic domain is defined in $[0,10] \times[0,10]$. A prescribed grid motion is employed in this case to validate the moving grid algorithm. The prescribed grid motion of an element vertex is defined $\mathrm{as}^{27}$

$$
\left\{\begin{array}{l}
x(t)=x_{r}+a_{x} \sin \left(f_{n} t\right) \sin \left(f_{x} x_{r}\right) \sin \left(f_{y} y_{r}\right) \\
y(t)=y_{r}+a_{y} \sin \left(f_{n} t\right) \sin \left(f_{x} x_{r}\right) \sin \left(f_{y} y_{r}\right)
\end{array}\right.
$$

where $x_{r}, y_{r}$ are the coordinates at $t=0$, which are regarded as reference coordinates for the grid deformation. In this study, $a_{x}=0.5, a_{y}=0.5, f_{n}=2.0 \pi, f_{x}=0.4 \pi$, and $f_{y}=0.4 \pi$. The grid velocities on solution points and flux points of one element are linearly interpolated from the analytical grid velocities of the four vertices of the current element, which can be expressed as

$$
\left\{\begin{array}{l}
x^{\prime}(t)=a_{x} f_{n} \cos \left(f_{n} t\right) \sin \left(f_{x} x_{r}\right) \sin \left(f_{y} y_{r}\right), \\
y^{\prime}(t)=a_{y} f_{n} \cos \left(f_{n} t\right) \sin \left(f_{x} x_{r}\right) \sin \left(f_{y} y_{r}\right) .
\end{array}\right.
$$

Periodic boundary conditions are applied to all boundaries. Simulations stop at $t=0.25$. The time step size $\Delta t=10^{-4}$ to make sure the errors are dominated by the spatial discretization. Grid refinement studies have been carried out on a uniform mesh set, i.e., $16 \times 16,32 \times 32$, and $64 \times 64$, for static meshes and deformable meshes. Figure 2 shows the density contour and deformable mesh at $t=0.25$. The grid refinement results are presented in Table 1 . It has been observed that the numerical orders of the CDFR methods tested here agree with the theoretical ones for both static meshes and dynamic meshes. The errors increase when the dynamic meshes are used.

\begin{tabular}{|c|c|c|c|c|c|c|}
\hline \multicolumn{7}{|c|}{ The grid refinement for the static meshes } \\
\hline & \multicolumn{2}{|l|}{ 3rd } & \multicolumn{2}{|l|}{4 th } & \multicolumn{2}{|l|}{5 th } \\
\hline Mesh & $E_{L_{2}}(\rho)$ & order & $E_{L_{2}}(\rho)$ & order & $E_{L_{2}}(\rho)$ & order \\
\hline $16 \times 16$ & $2.3345 \times 10^{-4}$ & & $2.5977 \times 10^{-5}$ & & $2.4706 \times 10^{-6}$ & \\
\hline $32 \times 32$ & $3.4760 \times 10^{-5}$ & 2.75 & $1.6803 \times 10^{-6}$ & 3.95 & $8.7390 \times 10^{-8}$ & 4.82 \\
\hline $64 \times 64$ & $4.4228 \times 10^{-6}$ & 2.97 & $1.0900 \times 10^{-7}$ & 3.95 & $2.7340 \times 10^{-9}$ & 5.00 \\
\hline \multicolumn{7}{|c|}{ The grid refinement for the dynamic meshes } \\
\hline Mesh & $E_{L_{2}}(\rho)$ & order & $E_{L_{2}}(\rho)$ & order & $E_{L_{2}}(\rho)$ & order \\
\hline $16 \times 16$ & $4.1983 \times 10^{-4}$ & & $6.1096 \times 10^{-5}$ & & $9.4836 \times 10^{-6}$ & \\
\hline $32 \times 32$ & $6.8795 \times 10^{-5}$ & 2.61 & $4.8656 \times 10^{-6}$ & 3.65 & $3.7677 \times 10^{-7}$ & 4.65 \\
\hline $64 \times 64$ & $9.6865 \times 10^{-6}$ & 2.83 & $3.4281 \times 10^{-7}$ & 3.83 & $1.4106 \times 10^{-8}$ & 4.74 \\
\hline
\end{tabular}

Table 1. The grid refinement studies of the 3rd-, 4th-, and 5th-order CDFR methods for the 2D isentropic Euler vortex propagation with and without dynamic meshes

\section{V.B. Couette Flow}

The Couette flow is studied to demonstrate the performance of the CDFR method for viscous flows in this section. The problem models the flow between two parallel plates, one of which is 


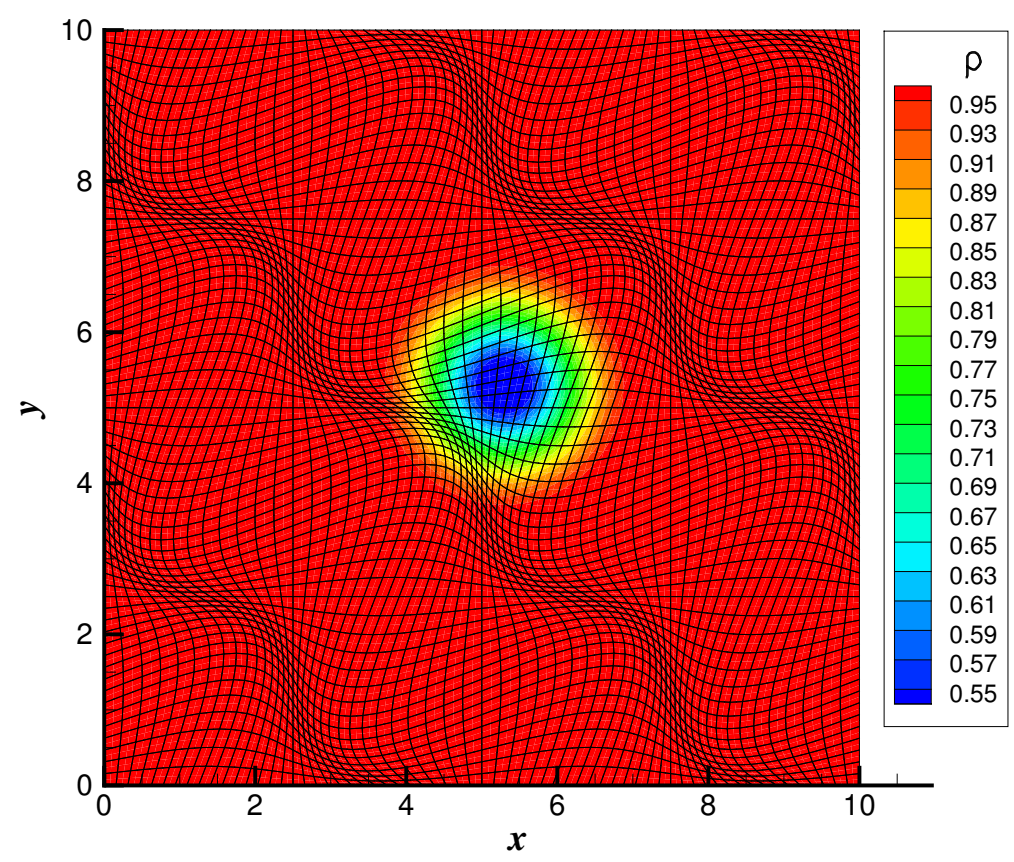

Figure 2. The deformable $64 \times 64$ mesh and the density contour of the 3rd-order CDFR method for the isentropic Euler vortex propagation case at $t=0.25$.

moving relative to the other in the tangential direction. In this study, one plate is fixed at $y=0$ while the other one at $y=H$ moves at the speed of $U$. The plate at $y=0$ has a temperature of $T_{0}$ while the other one has a temperature of $T_{1}$. The viscosity $\mu$ is a constant. The analytical solution for this problem can be expressed as ${ }^{33}$

$$
\left\{\begin{array}{l}
u=\frac{U}{H} y, \\
v=0 \\
T=T_{0}+\frac{y}{H}\left(T_{1}-T_{0}\right)+\frac{\mu U^{2}}{2 k} \frac{y}{H}\left(1-\frac{y}{H}\right), \\
\rho=\frac{p}{R T},
\end{array}\right.
$$

where $p=1.0$ is a constant in this study, $k$ is the thermal conductivity and $R=1.0$. The domain is $[0,4] \times[0,2]$. In the present study, $H=1, U=2, T_{0}=0.8, T_{1}=0.85$ and $\mu=0.01$. A mesh set, i.e., $10 \times 5,20 \times 10$ and $40 \times 20$, has been used for the grid refinement study of the 3rd-, 4th-, and 5th- order CDFR methods. A GMRES solver is used for the simulations to drive the residual to drop until $10^{-14}$. The grid refinement study results are presented in Table 2. The results demonstrates that the CDFR methods tested here can preserve the order of accuracy for the viscous flows.

Table 2. The grid refinement studies of the 3rd-, 4th-, and 5th-order CDFR methods for the Couette flow

\begin{tabular}{lllllll}
\hline \multicolumn{2}{c}{ 3rd } & \multicolumn{2}{c}{4 th } & \multicolumn{2}{c}{5 th } \\
\hline Mesh & $E_{L_{2}}(\rho)$ & order & $E_{L_{2}}(\rho)$ & order & $E_{L_{2}}(\rho)$ & order \\
\hline 540 & $2.1411 \times 10^{-6}$ & & $1.3647 \times 10^{-6}$ & & $4.5178 \times 10^{-9}$ & \\
2160 & $3.3966 \times 10^{-7}$ & 2.66 & $1.4128 \times 10^{-7}$ & 3.27 & $1.4272 \times 10^{-10}$ & 4.98 \\
8640 & $6.1362 \times 10^{-8}$ & 2.47 & $1.1354 \times 10^{-8}$ & 3.64 & $4.6144 \times 10^{-12}$ & 4.95 \\
\hline
\end{tabular}




\section{V.C. Laminar Flow Past a Circular Cylinder}

To validate the capability of the solver developed in this study, we first study the laminar unsteady flow of $M a=0.2$ and $R e=100$ past a static circular cylinder. The physical domain and the mesh are presented in Figure 3. The domain is the same size as that in Ref. 34, i.e., $(x, y) \in[-16,32] \times[-16,16]$. There are 32 elements around the circumference of the cylinder and 40 elements along the normal direction. The first layer of the mesh is about $10 \%$ of the radius of the cylinder. $P^{4}$ elements are employed to accurately represent the wall boundaries. The Dirichlet boundary condition of $\left(\rho_{\infty}, u_{\infty}, 0, p_{\infty}\right)$ is applied to the left boundary and for the right boundary the fixed-pressure boundary condition is employed. The top and bottom boundaries are treated as symmetric surfaces. A fifth order CDFR method is used for the simulation. The time step size $\Delta t$ is $2 \times 10^{-4}$ for the SSPRK3. The drag coefficient $\overline{C_{d}}$, root mean square (RMS) of the lift coefficient $C_{l, R M S}$, and Strouhal number $S t$ are presented in Table 3. Through the comparison with previous studies, it is validated that the CDFR method can predict the correct physics for the laminar flow past a static circular cylinder. The vorticity field in the wake region at $t=200$ is presented in Figure 4 . On this relative coarse mesh adopted in the simulation, the CDFR method has a good resolution of the vorticity field at about 14 times of the diameter of cylinder in the wake region.

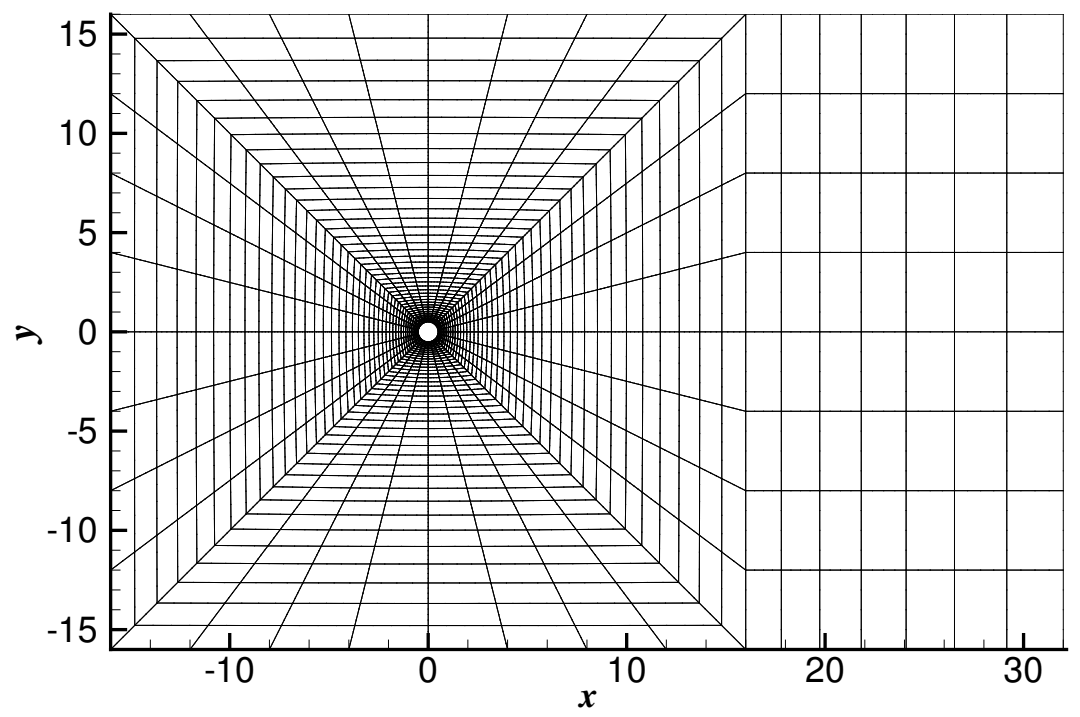

Figure 3. An illustration of the physical domain and mesh for the flow of $M a=0.2$ and $R e=100$ past a circular cylinder.

Table 3. A comparison of the present lift and drag results of the laminar flow of $R e=100$ and $M a=0.2$ past a circular cylinder with previous studies

\begin{tabular}{llll}
\hline & Present study & Liang et al.(2009) & Meneghini \& Saltara $(2001)^{35}$ \\
\hline$\overline{C_{d}}$ & 1.367 & 1.365 & 1.37 \\
$C_{l, R M S}$ & 0.231 & 0.232 & \\
$S t$ & 0.165 & 0.164 & 0.165 \\
\hline
\end{tabular}

We have also simulated the laminar flow of $R e=185$ and $M a=0.2$ past a static and oscillating circular cylinder in this study. For this case a larger physical domain $((x, y) \in$ $[140 \sqrt{2}, 140 \sqrt{2}] \times[140 \sqrt{2}, 140 \sqrt{2}])$ is considered, which is presented in Figure 5 with the mesh. There are 40 elements around the circumference of the cylinder and 60 elements along the normal direction. The mesh in the wake region is refined compared to that of the previous case while other mesh set-up in $[-16,16] \times[-16,16]$ is the same. For this case, Riemann solvers are 


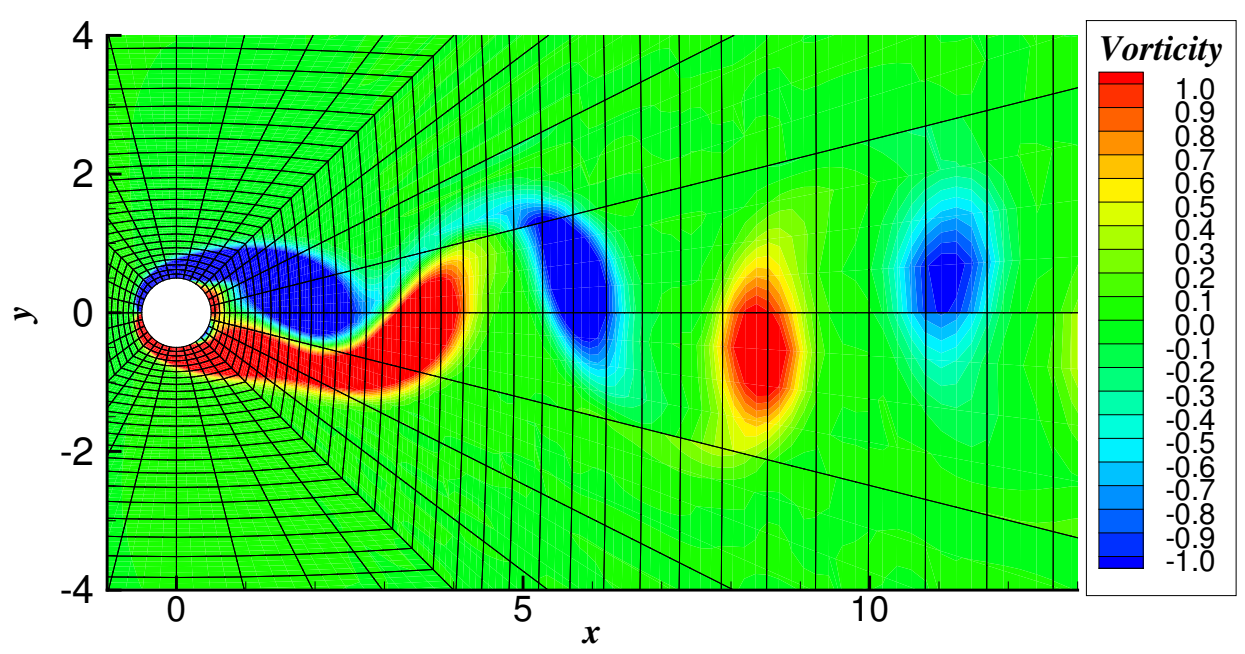

Figure 4. The vorticity field in the weak region at $t=200$. The laminar flow is of $R e=100$ and $M a=0.2$.

employed on all boundaries to loosely apply the Dirichlet boundary condition $\left(\rho_{\infty}, u_{\infty}, 0, p_{\infty}\right)$ to the inner domain. The spatial discretization is the same as the previous case as well as the time integration and the time step size. We report the force results in Table 4. And Figure 7 gives the $C_{d}$ and $C_{l}$ history. The vorticity field in the wake region at $t=200$ is presented in Figure 6. The current study is compared to an incompressible laminar flow of $R e=185$ past a circular cylinder reported in Refs. 28,36 . The compressibility will slightly increase the $\overline{C_{d}}$ by less than $2 \%$ and will only have a trivial effect on the $C_{l, R M S}$. The Strouhal number $S t$ is the same. This phenomenon has also been reported in Ref. 34 .

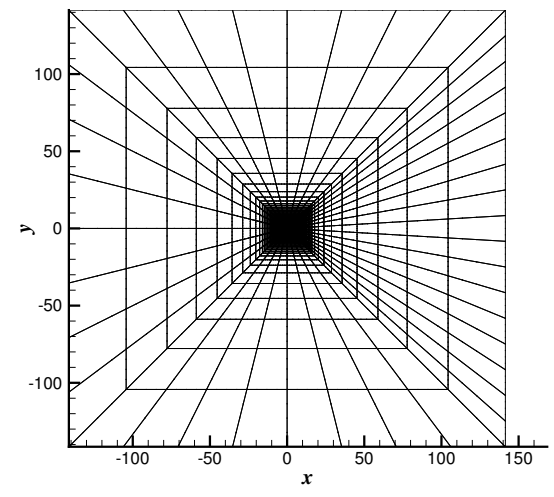

(a)

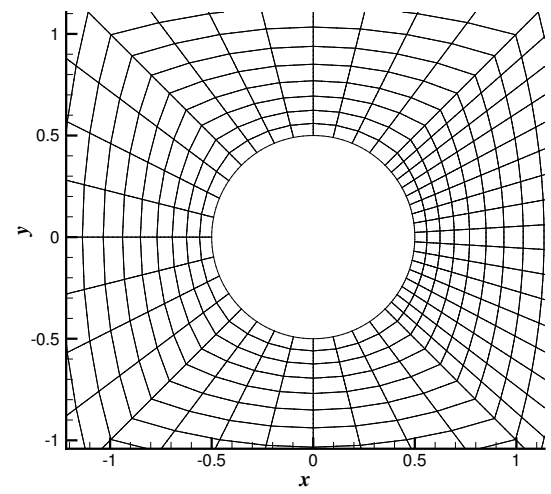

(b)

Figure 5. (a) The domain and mesh for the flow of $R e=180$ and $M a=0.2$ and (b) an enlarged view of the mesh close to the circular cylinder.

When the cylinder oscillates perpendicular to the incoming flow, we select three different oscillating frequencies in this study. The rigid-body motion defined as

$$
y=A \sin (2 \pi f t)
$$

is considered, where $A=0.2 D$ is the amplitude and $D$ is the diameter of the cylinder. The oscillating frequency is defined by the frequency ratio $r=f / f_{0}$, where $f_{0}$ is the natural vortex shedding ratio. $f_{0}=0.192$ is obtained from the previous study. Herein, we study the flow physics when $r=0.8, r=1.0$ and $r=1.2$. The $C_{d}$ and $C_{l}$ histories are presented in Figure 8, Figure 9 and Figure 10, recpectively. We have observed the similar phenomena as Ref. 28,37, even though 
Table 4. A comparison of the present lift and drag results of the laminar flow of $R e=185$ and $M a=0.2$ past a circular cylinder with previous studies

\begin{tabular}{lllll}
\hline & Present study & Yu \& Wang $(2016)^{28}$ & Lu \& Dalton(1996) & Guilmineau \&Queutey $^{37}$ \\
\hline$\overline{C_{d}}$ & 1.341 & 1.317 & 1.31 & 1.287 \\
$C_{l, R M S}$ & 0.446 & 0.441 & 0.442 & 0.443 \\
$S t$ & 0.192 & 0.192 & 0.195 & 0.195 \\
\hline
\end{tabular}

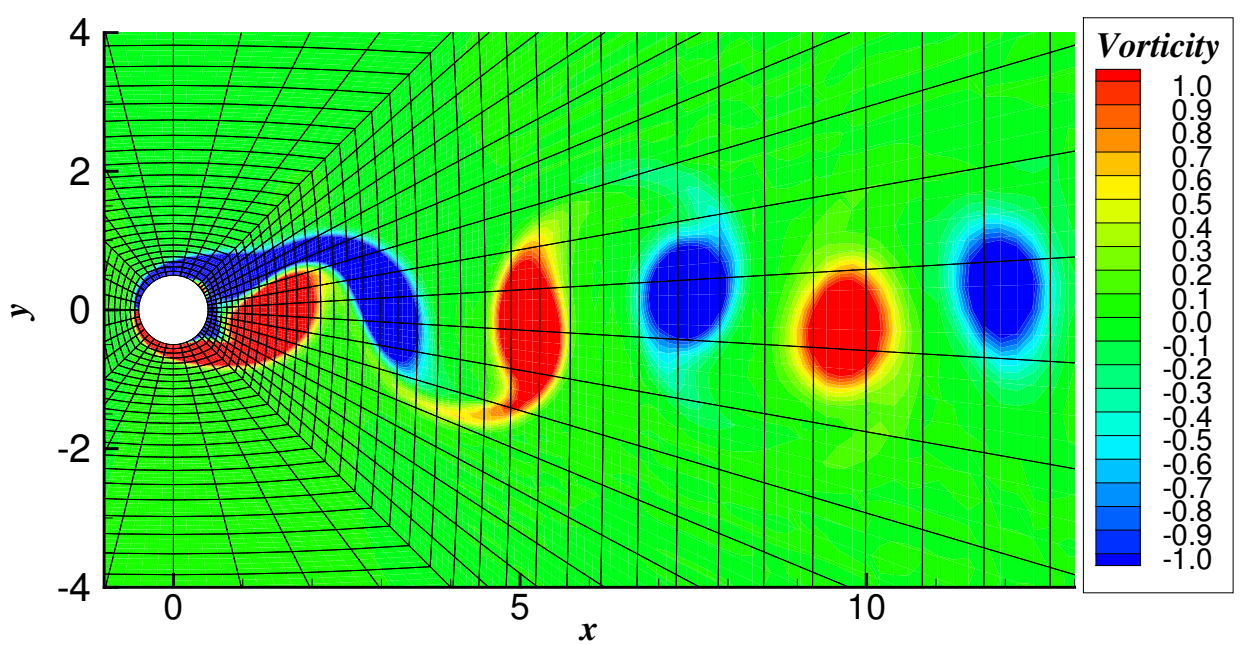

Figure 6. The vorticity field in the weak region at $t=200$. The laminar flow is of $R e=185$ and $M a=0.2$.

the weak compressibility affects the forces slightly. When the frequency of the excitation does not exceed the natural vortex shedding frequency $f_{0}$, the flow will still exhibit periodic features. When the excitation frequency exceeds the natural vortex shedding frequency. Chaotic features will be observed. This observation agrees with the studies conducted in Refs. 28,37.

\section{Conclusions}

In this study, the CDFR method has been further developed to solve the 2D Navier-Stokes equations with dynamic meshes. The GCL is enforced analytically in the transformation of the governing equations from the physical space-time domain to the computational space-time domain. The SSPRK3 method is employed for unsteady problems coupled with dynamic meshes. We have verified the accuracy of the CDFR method through various convergence studies, including inviscid and viscous problems. With the explicit time integration, we have also demonstrate that the GCL is enforced to guarantee that the numerical orders of accuracy are not affected by grid motions. Laminar flows $(R e=100$ and $R e=185)$ past a circular cylinder have been studied. Through comparison with previous studies in literatures, it is demonstrated that the CDFR method can predict the forces and flow physics accurately for unsteady problems involving dynamic meshes.

\section{Acknowledgment}

The authors gratefully acknowledge the support of the Office of Naval Research through the award N00014-16-1-2735, and the faculty startup support from the department of mechanical engineering at University of Maryland, Baltimore County (UMBC). 


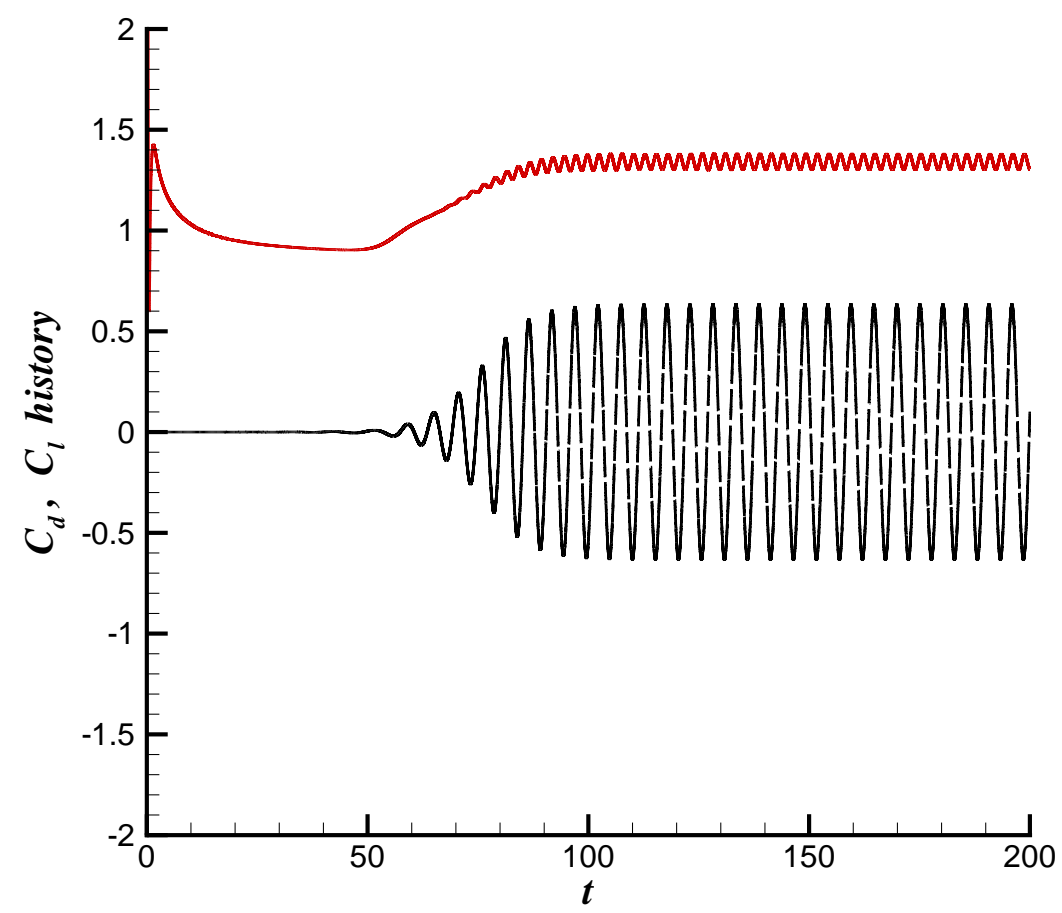

Figure 7. The $C_{d}$ and $C_{l}$ history of the laminar flow of $R e=185$ and $M a=0.2$ past a static cylinder.

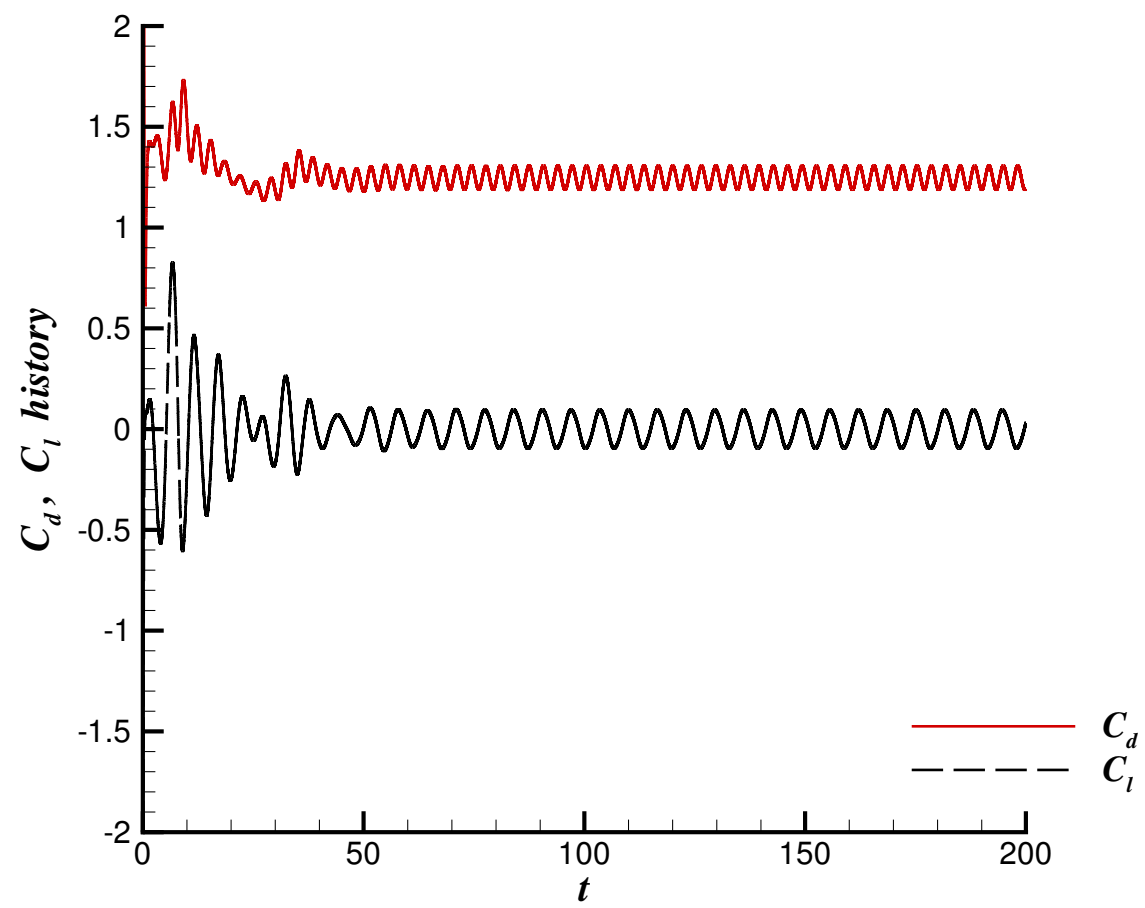

Figure 8. The $C_{d}$ and $C_{l}$ history of the laminar flow of $R e=185$ and $M a=0.2$ past an oscillating cylinder. The excitation frequency ratio is 0.8 . 


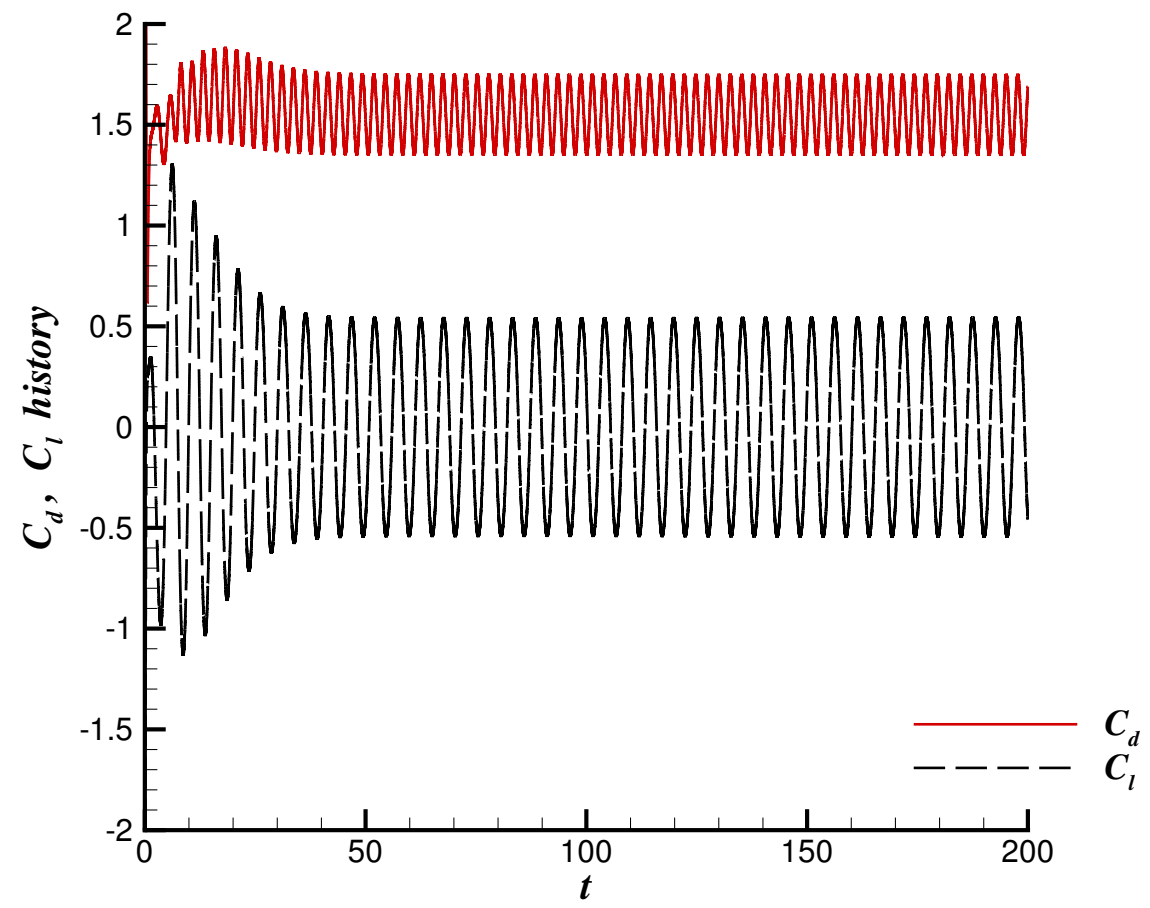

Figure 9. The $C_{d}$ and $C_{l}$ history of the laminar flow of $R e=185$ and $M a=0.2$ past an oscillating cylinder. The excitation frequency ratio is 1.0.

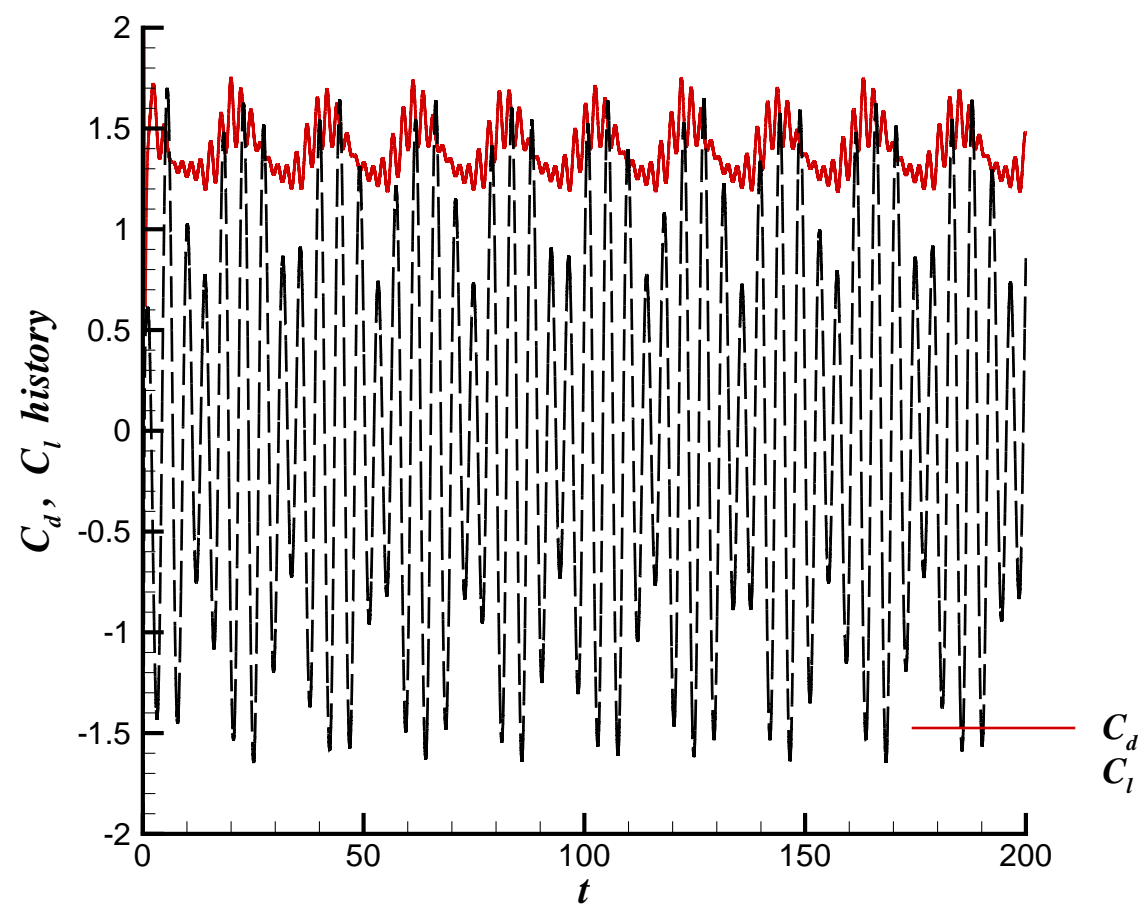

Figure 10. The $C_{d}$ and $C_{l}$ history of the laminar flow of $R e=185$ and $M a=0.2$ past an oscillating cylinder. The excitation frequency ratio is $\mathbf{1 . 2}$. 


\section{References}

${ }^{1}$ J. A. Ekaterinaris, "High-order accurate, low numerical diffusion methods for aerodynamics," Progress in Aerospace Sciences, vol. 41, pp. 192-300, 1998.

${ }^{2}$ Z. J. Wang, "High-order methods for the Euler and Navier-Stokes equations on unstructured grids," Progress in Aerospace Sciences, vol. 43, pp. 1-41, 2007.

${ }^{3}$ Z. J. Wang, K. Fidkowski, R. Abgrall, F. Bassi, D. Caraeni, A. Cary, H. Deconinck, R. Hartmann, K. Hillewaert, H. T. Huynh, N. Kroll, G. May, P.-O. Persson, B. van Leer and M. Visbal, "High-order CFD methods: current status and perspective," International Journal for Numerical Methods in Fluids, vol. 72, pp. 811-845, 2013.

${ }^{4}$ S. K. Lele, "Compact finite difference schemes with spectral-like resolution," Journal of Computational Physics, vol. 103, pp. 16-42, 1992.

${ }^{5}$ X. Liu, S. Osher and T. Chan, "Weighted essentially non-oscillatory schemes," Journal of Computational Physics, vol. 115, pp. 200-212, 1994.

${ }^{6}$ G.-S. Jiang and C.-W. Shu, "Efficient implementation of weighted ENO schemes," Journal of Computational Physics, vol. 126, pp. 202-228, 1996.

${ }^{7}$ B. Cockburn and C.-W. Shu, "TVB Runge-Kutta local projection discontinuous Galerkin finite element method for conservation laws II: general framework," Mathematics of Computation, vol. 52, pp. 411-435, 1989.

${ }^{8}$ F. Bassi and S. Rebay, "High-order accurate discontinuous finite element solution of the 2D Euler equations," Journal of Computational Physics, vol. 138, pp. 251-285, 1997.

${ }^{9}$ Z. J. Wang, "Spectral (finite) volume method for conservation laws on unstructured grids: basic formulation," Journal of Computational Physics, vol. 178, pp. 210-251, 2002.

${ }^{10}$ D. A. Kopriva and J. H. Kolias, "A conservative staggered-grid Chebyshev multidomain method for compressible flows," Journal of Computational Physics, vol. 125, pp. 244-261, 1996.

${ }^{11}$ Y. Liu, M. Vinokur and Z. J. Wang, "Spectral difference method for unstructured grids I: Basic formulation," Journal of Computational Physics, vol. 216, pp. 780-801, 2006.

${ }^{12} \mathrm{H}$. T. Huynh, "A flux reconstruction approach to high-order schemes including discontinuous Galerkin methods," in the 18th AIAA Computational Fluid Dynamics Conference, (Miami, FL), 2007. AIAA-2007-4079.

${ }^{13} \mathrm{H}$. T. Huynh, "A reconstruction approach to high-order schemes including discontinuous Galerkin methods for diffusion," in the 47th AIAA Aerospace Sciences Meeting including The New Horizons Forum and Aerospace, (Orlando, FL), 2007. AIAA-2009-403.

${ }^{14}$ Z. J. Wang and H. Y. Gao, "A unifying lifting collocation penalty formulation including the discontinuous Galerkin, spectral volume/difference methods for conservation laws on mixed grids," Journal of Computational Physics, vol. 228, p. 8161-8186, 2009.

${ }^{15}$ P. E. Vincent, P. Castonguay and A. Jameson, "A new class of high-order energy stable flux reconstruction schemes," Journal of Scientific Computing, vol. 47, pp. 50-72, 2011.

${ }^{16}$ M. H. Carpenter, D. Gottlieb and S. Abarbanel, "The Stability of Numerical Boundary Treatments for Compact High-Order Finite-Difference Schemes," Journal of Computational Physics, vol. 108, pp. 272-295, 1993.

${ }^{17}$ D. Ghosh and J. Baeder, "Compact reconstruction schemes with weighted ENO limiting for hyperbolic conservation laws," SIAM Journal on Scientific Computing, vol. 34, pp. A1678-A1706, 2012.

${ }^{18}$ S. Pirozzoli, "Conservative hybrid compact-WENO schemes for shock-turbulence interaction," Journal of Computational Physics, vol. 178, pp. 81-117, 2002.

${ }^{19}$ M. Svard and J. Nordström, "Review of summation-by-parts schemes for initial-boundary-value problems," Journal of Computational Physics, vol. 268, pp. 17-38, 2014.

${ }^{20} \mathrm{~J}$. Nordström and M. H. Carpenter, "Boundary and Interface Conditions for High-Order Finite-Difference Methods Applied to the Euler and Navier-Stokes Equations," Journal of Computational Physics, vol. 148, pp. 621-645, 1999.

${ }^{21}$ T. C. Fisher, M. H. Carpenter, J Nordström, N. K. Yamaleev and C. Swanson, "Discretely conservative finite-difference formulations for nonlinear conservation laws in split form: Theory and boundary conditions," Journal of Computational Physics, vol. 231, pp. 353-375, 2013.

${ }^{22}$ H. T. Huynh, Z. J. Wang and P. E. Vincent, "High-order methods for computational fluid dynamics: A brief review of compact differential formulations on unstructured grids," Computers \& Fluids, vol. 98, pp. 209-220, 2014.

${ }^{23}$ J. Romero, K. Asthana and A. Jameson, "A simplified formulation of the flux reconstruction method," Journal of Scientific Computing, vol. 67, pp. 351-372, 2016.

${ }^{24} \mathrm{~J}$. Romero and A. Jameson, "Extension of the flux reconstruction method to triangular elements using collapsed-edge quadrilaterals," in the 54th AIAA Aerospace Sciences Meeting, (San Diego, CA), 2016. AIAA2016-1825.

${ }^{25}$ L. Wang and M. L. Yu, "Compact Direct Flux Reconstruction for Conservation Laws," resubmitted after revision. 
${ }^{26}$ M. R. Visbal and D. V. Gaitonde, "On the use of higher-order finite-difference schemes on curvilinear and deforming meshes," Journal of Computational Physics, vol. 181, pp. 155-185, 2002.

${ }^{27}$ M. L. Yu, Z. J. Wang and H. Hu, "A high-order spectral difference method for unstructured dynamic grids," Computers 85 Fluids, vol. 48, pp. 84-97, 2011.

${ }^{28}$ M. L. Yu and L. Wang, "A high-order flux reconstruction/correction procedure via reconstruction formulation for unsteady incompressible flow on unstructured moving grids," Computers \& Fluids, vol. 139, pp. 161-173, 2016.

${ }^{29}$ P.-O. Persson, J. Bonet and J. Peraire, "Discontinuous Galerkin solution of the Navier-Stokes equations on deformable domains," Computer Methods in Applied Mechanics and Engineering, vol. 198, pp. 1585-1595, 2009.

${ }^{30}$ P. L. Roe, "Approximate Riemann solvers, parameter vectors and difference schemes," Journal of Computational Physics, vol. 43, pp. 357-372, 1981.

${ }^{31}$ S. Gottlieb, C.-W. Shu and E. Tadmor, "Strong stability-preserving high-order time discretization methods," SIAM review, vol. 43, pp. 89-112, 2001.

${ }^{32}$ F. Bassi, L. Botti, A. Colombo, A Ghidoni and F. Massa, "Linearly implicit Rosenbrock-type Runge-Kutta schemes applied to the Discontinuous Galerkin solution of compressible and incompressible unsteady flows," Computers $\&$ Fluids, vol. 118, pp. 305-320, 2015.

${ }^{33}$ Y. Z. Sun, Z. J. Wang and Y. Liu, "High-order multidomain spectral difference method for the Navier-Stokes Equations on Unstructured Hexahedral Grids," Communications in Computational Physics, vol. 2, pp. 310-333, 2007.

${ }^{34}$ C. L. Liang, S. Premasuthan and A. Jameson, "High-order accurate simulation of low-Mach laminar flow past two side-by-side cylinders using spectral difference method," Computers and Structures, vol. 87, pp. 812-827, 2009 .

${ }^{35}$ J. R. Meneghini, F. Saltara, C. L. R. Siqueira and J. A. Ferrari, "Numerical simulation of flow interference between tow circular cylinders in tandem and side-by-side arrangement," Journal of Fluids and Structures, vol. 15, pp. 327-350, 2001.

${ }^{36}$ X. Y. Lu and C. Dalton, "Calculation of the timing of vortex formation from an oscillating cylinder," Journal of Fluids and Structures, vol. 10, pp. 527-541, 1996.

${ }^{37}$ E. Guilmineau and P. Queutey, "A numerical simulation of vortex shedding from an oscillating cylinder," Journal of Fluids and Structures, vol. 16, pp. 773-794, 2002. 